\title{
Interaction of vector mesons with baryons and nuclei
}

\author{
E. Oset ${ }^{1}$, A. Ramos ${ }^{2}$, E. J. Garzon ${ }^{1}$, R. Molina ${ }^{3}$, L. \\ Tolos $^{4}, 5$, C. W. Xiao ${ }^{1}$, J. J. $\mathrm{Wu}^{6}$ and B. S. Zou ${ }^{7,8}$ \\ ${ }^{1}$ Departamento de Física Teórica and IFIC, \\ Centro Mixto Universidad de Valencia-CSIC, \\ Institutos de Investigación de Paterna, \\ Aptdo. 22085, 46071 Valencia, Spain \\ 2 Departament d'Estructura $i$ Constituents de la Materia, Universitat de Barcelona \\ ${ }^{3}$ Research Center for Nuclear Physics (RCNP), \\ Mihogaoka 10-1, Ibaraki 567-0047, Japan \\ ${ }^{4}$ Instituto de Ciencias del Espacio (IEEC/CSIC) Campus Universitat Autònoma de Barcelona, \\ Facultat de Ciències, Torre C5, E-08193 Bellaterra (Barcelona), Spain \\ ${ }^{5}$ Frankfurt Institute for Advanced Studies (FIAS). Johann Wolfgang Goethe \\ University. Ruth-Moufang-Str. 1. 60438 Frankfurt am Main. Germany \\ ${ }^{6}$ Physics Division, Argonne National Laboratory, Argonne, Illinois 60439, USA \\ ${ }^{7}$ Institute of High Energy Physics, CAS, \\ P.O.Box 918(4), Beijing 100049, China \\ ${ }^{8}$ Theoretical Physics Center for Science Facilities, CAS, Beijing 100049, China
}

(Dated: October 16, 2012)

\begin{abstract}
After some short introductory remarks on particular issues on the vector mesons in nuclei, in this paper we present a short review of recent developments concerning the interaction of vector mesons with baryons and with nuclei from a modern perspective using the local hidden gauge formalism for the interaction of vector mesons. We present results for the vector baryon interaction and in particular for the resonances which appear as composite states, dynamically generated from the interaction of vector mesons with baryons, taking also the mixing of these states with pseudoscalars and baryons into account. We then venture into the charm sector, reporting on hidden charm baryon states around $4400 \mathrm{MeV}$, generated from the interaction of vector mesons and baryons with charm, which have a strong repercussion on the properties of the $J / \Psi N$ interaction. We also address the interaction of $K^{*}$ with nuclei and make suggestions to measure the predicted huge width in the medium by means of the transparency ratio. The formalism is extended to study the phenomenon of $J / \psi$ suppression in nuclei via $J / \psi$ photoproduction reactions.
\end{abstract}

PACS numbers: $11.80 . \mathrm{Gw}, 12.38 . \mathrm{Gc}, 12.39 . \mathrm{Fe}, 13.75 . \mathrm{Lb}$ 


\section{INTRODUCTION}

The topic of vector meson interactions with nuclei has attracted, and continues to attract, much attention. After thorough experimental and theoretical studies of pion nuclear interaction and other pseudoscalar nucleus interactions, the turn came for exploring the properties of the vector mesons in nuclei. In this limited study we do not pretend to make an exhaustive review of the field, which has been done anyway in other papers [1] and more recently in [2, 3. . We shall make emphasis on the new perspective that the use of the local hidden gauge theory [4 [6] has brought into this topic.

From a historical perspective, one certainly must admit, that in spite of much theoretical evidence against it from detailed calculations [7-12], the guess in [13] that the vector meson masses would be drastically reduced in a nuclear medium stimulated much experimental work. Experimental searches have finally concluded that this was not the case [2, 3, 14, 15]. However, a few surprises have been found on the way.

As a matter of example let us recall here the history concerning the $\omega$ mass in the medium from experiments in the ELSA (Bonn) Laboratory. The analysis of the experimental results on the photoproduction of $\omega$, observed from the decay channel $\pi^{0} \gamma$, led the authors of [16] to claim the first observation of in-medium modifications of the $\omega$ meson mass, by an approximate amount of $100 \mathrm{MeV}$ for normal nuclear matter density. Yet, the observation in [17] that the results of [16] were tied to a particular choice of background led to a thorough search for background processes in [18] that finished with the withdrawal of the $\omega$ mass shift claims. In between, suggestions that some signal reported in [19, 20] could be indicative of an $\omega$ meson bound state in nuclei were aborted very early, realizing that a double hump structure in the experiment was due to a different scaling of the uncorrelated $\pi^{0} \gamma$ production events and the $\omega$ production process with subsequent $\pi^{0} \gamma$ decay [21]. Before the final conclusions of [18], it was also hoped that the use of the successful mixed events method to separate the background from the signal should be sufficient to isolate the $\omega$ signal [22]. However, a simulation of the reaction [24] showed that the mixed event method produced a background essentially independent of the real background in the region of relevance to omega production, basically determined from events occurring at much lower invariant masses.

The persistence of both theoretical and experimental teams in the clarification of the problem gave undoubtedly some fruits and this is now the problem most thoroughly studied in this field, that has led to clarifications in other related problems. Yet, there is some interesting physical information that survived the close scrutiny of the former works and this is the large width of the $\omega$ in the medium found in [23] and also studied in [17]. The width of the $\omega$ in the medium extrapolated to normal nuclear matter density was of the order of $100 \mathrm{MeV}$ in [17] and 130-150 $\mathrm{MeV}$ in [23]. The theoretical understanding of this large width, related to decay channels of the $\omega$ in the nuclear medium, is a challenge that will require combined efforts of hadron dynamics and many body theory.

In this paper we shall review recent developments on the interaction of vector mesons with baryons and nuclei, using effective field theory with a combination of effective Lagrangians to account for hadron interactions, and implementing exactly unitarity in coupled channels. This approach is a very efficient tool to face many problems in Hadron Physics. Using this coupled channel unitary approach with the input from chiral Lagrangians, usually referred to as chiral unitary approach, the interaction of the octet of pseudoscalar mesons with the octet of stable baryons has been studied and leads to $J^{P}=1 / 2^{-}$resonances which fit quite well the spectrum of the known low lying resonances with these quantum numbers [25 30]. Among the new resonances predicted, the most notable is the $\Lambda(1405)$, where all the chiral approaches find two poles close by [31 37], rather than one, for which experimental support is presented in [38, 39]. Another step forward in this direction has been the interpretation of low lying $J^{P}=1 / 2^{+}$states as molecular systems of two pseudoscalar mesons and one baryon [40 44]. 
More recently, vectors instead of pseudoscalars have also been considered. In the baryon sector the interaction of the $\rho \Delta$ interaction was addressed in [45], where three degenerate $N^{*}$ states and three degenerate $\Delta$ states around $1900 \mathrm{MeV}$, with $J^{P}=1 / 2^{-}, 3 / 2^{-}, 5 / 2^{-}$, were found. The extrapolation to $\mathrm{SU}(3)$ with the interaction of the vectors of the nonet with the baryons of the decuplet was studied in [46]. The starting point of these works is the hidden gauge formalism [4 6], which deals with the interaction of vector mesons and pseudoscalars, respecting chiral dynamics, providing the interaction of pseudoscalars among themselves, with vector mesons, and vector mesons among themselves. It also offers a perspective on the chiral Lagrangians as limiting cases at low energies of vector exchange diagrams occurring in the theory.

The results of the interaction of the nonet of vector mesons with the octet of baryons were reported in [47]. The scattering amplitudes, obtained under the approximation of neglecting the three momentum of the particles versus their mass, led to poles in the complex plane which can be associated to some well known resonances. In [47] one obtains degenerate states of $J^{P}=1 / 2^{-}, 3 / 2^{-}$, a degeneracy that seems to be followed qualitatively by the experimental spectrum, although in some cases the spin partners have not been identified. We will also report on improvements in this theory which consider the mixing of the vector-baryon states with pseudoscalar-baryon ones.

In fact, there is in principle no reason to expect that the interaction of pseudoscalar mesons with baryons and the interaction of vector mesons with baryons should be decoupled for states which share strangeness, isospin, and $J^{P}$ (spin-parity) quantum numbers. The consequences of coupling these interactions, that have been treated independently in the previous works of [46] and [47], were first explored in the three flavour sector in Ref. [48]. In that work, an SU(6) framework [49 51. was used which combines spin and flavor symmetries within an enlarged Weinberg-Tomozawa meson-baryon Lagrangian in order to accommodate vector mesons and decuplet baryons. This guarantees that chiral symmetry is recovered when interactions involving pseudoscalar NambuGoldstone bosons are being examined ${ }^{1}$. Chiral symmetry constraints the pseudoscalar octet-baryon decuplet interactions. However, the interaction of vector mesons with baryons is not constrained by chiral symmetry, and thus the model presented in [48] differs from those of Refs. [46, 47], based on the hidden gauge formalism. However, in the presence of heavy quarks the analogous scheme to that of Ref. [48] automatically embodies heavy quark spin symmetry, another well established approximate symmetry of QCD. Indeed, the model of Ref. [48] has been successfully extended to the charm sector in 53 55].

The vast number of resonances with charm or hidden charm found in the recent years, some of which having a clear molecular structure, and the possibility of studying them copiously at LHC or upgraded B-factories, has injected a renewed interest in this field. We will report on composite states of hidden charm emerging from the interaction of vector mesons and baryons with charm.

Finally, we devote some attention to new developments on the properties of vector mesons in a nuclear medium, focusing specifically on the interactions of the $K^{*}$ and $J / \psi$ mesons with nuclei.

\section{FORMALISM FOR $V V$ INTERACTION}

The formalism of the hidden gauge interaction for vector mesons is provided in [4, 5] (see also [56] for a practical set of Feynman rules). The Lagrangian involving the interaction of vector mesons amongst themselves is given by

$$
\mathcal{L}_{I I I}=-\frac{1}{4}\left\langle V_{\mu \nu} V^{\mu \nu}\right\rangle
$$

\footnotetext{
${ }^{1}$ A similar study for the case of meson-meson light resonances was carried out in Ref. [52].
} 

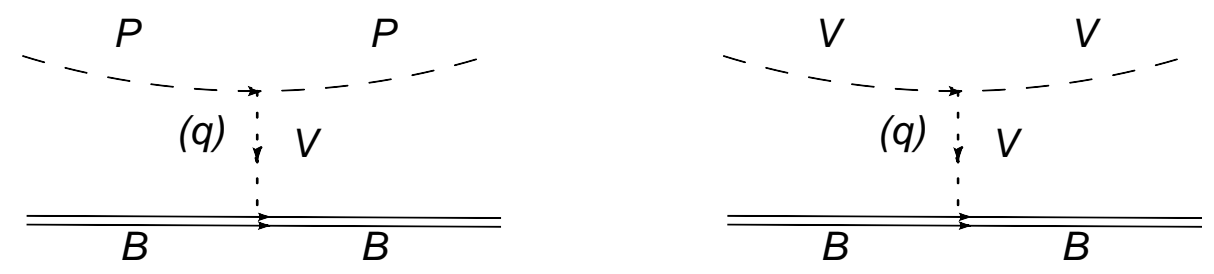

FIG. 1: Diagrams obtained in the effective chiral Lagrangians for interaction of pseudoscalar [a] or vector [b] mesons with the octet or decuplet of baryons.

where the symbol \langle\rangle stands for the trace in the $\mathrm{SU}(3)$ space and $V_{\mu \nu}$ is given by

$$
V_{\mu \nu}=\partial_{\mu} V_{\nu}-\partial_{\nu} V_{\mu}-i g\left[V_{\mu}, V_{\nu}\right]
$$

with $g$ given by $g=\frac{M_{V}}{2 f}$ where $f=93 \mathrm{MeV}$ is the pion decay constant. The magnitude $V_{\mu}$ is the $\mathrm{SU}(3)$ matrix of the vectors of the nonet of the $\rho$

$$
V_{\mu}=\left(\begin{array}{ccc}
\frac{\rho^{0}}{\sqrt{2}}+\frac{\omega}{\sqrt{2}} & \rho^{+} & K^{*+} \\
\rho^{-} & -\frac{\rho^{0}}{\sqrt{2}}+\frac{\omega}{\sqrt{2}} & K^{* 0} \\
K^{*-} & \bar{K}^{* 0} & \phi
\end{array}\right)_{\mu} .
$$

The interaction of $\mathcal{L}_{I I I}$ gives rise to a contact term coming from $\left[V_{\mu}, V_{\nu}\right]\left[V_{\mu}, V_{\nu}\right]$

$$
\mathcal{L}_{I I I}^{(c)}=\frac{g^{2}}{2}\left\langle V_{\mu} V_{\nu} V^{\mu} V^{\nu}-V_{\nu} V_{\mu} V^{\mu} V^{\nu}\right\rangle
$$

as well as to a three vector vertex from

$$
\mathcal{L}_{I I I}^{(3 V)}=i g\left\langle\left(\partial_{\mu} V_{\nu}-\partial_{\nu} V_{\mu}\right) V^{\mu} V^{\nu}\right\rangle=i g\left\langle\left(V^{\mu} \partial_{\nu} V_{\mu}-\partial_{\nu} V_{\mu} V^{\mu}\right) V^{\nu}\right\rangle .
$$

It is worth stressing the analogy with the coupling of vectors to pseudoscalars given in the same theory by

$$
\mathcal{L}_{V P P}=-i g\left\langle\left[P, \partial_{\mu} P\right] V^{\mu}\right\rangle,
$$

where $P$ is the $\mathrm{SU}(3)$ matrix of the pseudoscalar fields.

The Lagrangian for the coupling of vector mesons to the baryon octet is given by [57, 58]

$$
\mathcal{L}_{B B V}=\frac{g}{2}\left(\left\langle\bar{B} \gamma_{\mu}\left[V^{\mu}, B\right]\right\rangle+\left\langle\bar{B} \gamma_{\mu} B\right\rangle\left\langle V^{\mu}\right\rangle\right),
$$

where $B$ is now the $\mathrm{SU}(3)$ matrix of the baryon octet [59, 60]. Similarly, one has also a Lagrangian for the coupling of the vector mesons to the baryons of the decuplet, which can be found in [61].

Starting from these Lagrangians one can draw the Feynman diagrams that lead to the $P B \rightarrow P B$ and $V B \rightarrow V B$ interaction, by exchanging a vector meson between the pseudoscalar or the vector meson and the baryon, as depicted in Fig.1.

It was shown in [47] that in the limit of small three momenta of the vector mesons the vertices of Eq. (5) and Eq. (6) give rise to the same expression. This makes the work technically easy, allowing the use of many previous results.

A caveat must be made in the case of vector mesons due to the mixing of $\omega_{8}$ and the singlet of $\mathrm{SU}(3), \omega_{1}$, to give the physical states of the $\omega$ and the $\phi$. In this case, all one must do is to take the 
matrix elements known for the $P B$ interaction and, wherever $P$ is the $\eta_{8}$, multiply the amplitude by the factor $1 / \sqrt{3}$ to get the corresponding $\omega$ contribution and by $-\sqrt{2 / 3}$ to get the corresponding $\phi$ contribution. Upon the approximation consistent with neglecting the three momentum versus the mass of the particles (in this case the baryon), we can just take the $\gamma^{0}$ component of Eq. (7) and then the transition potential corresponding to the diagram of Fig. 1(b) is given by

$$
V_{i j}=-C_{i j} \frac{1}{4 f^{2}}\left(k^{0}+k^{\prime 0}\right) \overrightarrow{\epsilon \epsilon}^{\prime},
$$

where $k^{0}, k^{\prime 0}$ are the energies of the incoming and outgoing vector mesons. The same occurs in the case of the decuplet. The $C_{i j}$ coefficients of Eq. (8) can be obtained directly from [26, 62, 63] with the simple rules given above for the $\omega$ and the $\phi$, and substituting $\pi$ by $\rho$ and $K$ by $K^{*}$ in the matrix elements.

The scattering matrix is constructed by solving the coupled channels Bethe Salpeter equation in the on shell factorization approach of [26, 27]

$$
T=[1-V G]^{-1} V
$$

with $G$ being the loop function of a vector meson and a baryon, which we calculate in dimensional regularization using the formula of [27] and similar values for the subtraction constants. In the present case the $\rho$ and the $K^{*}$ have a significant width and the $G$ functions involving these mesons must be convoluted with their corresponding spectral functions.

The iteration of diagrams implicit in the Bethe Salpeter equation in the case of the vector mesons propagates the $\vec{\epsilon} \vec{\epsilon}^{\prime}$ term of the interaction. Hence, the factor $\vec{\epsilon} \vec{\epsilon}^{\prime}$ appearing in the potential $V$ factorizes also in the $T$ matrix for the external vector mesons. As a consequence of this, the interaction is spin independent and one finds degenerate states having $J^{P}=1 / 2^{-}$and $J^{P}=3 / 2^{-}$.

\section{INCORPORATING THE PSEUDOSCALAR MESON-BARYON CHANNELS}

Improvements to the work of [47] have been done by incorporating intermediate states of a pseudoscalar meson and a baryon in [64]. This is practically implemented by including the diagrams of Fig. (2). However, arguments of gauge invariance [7-12, 65, 66] demand that the meson pole term be accompanied by the corresponding Kroll-Ruderman contact term, see Fig. 3 ,

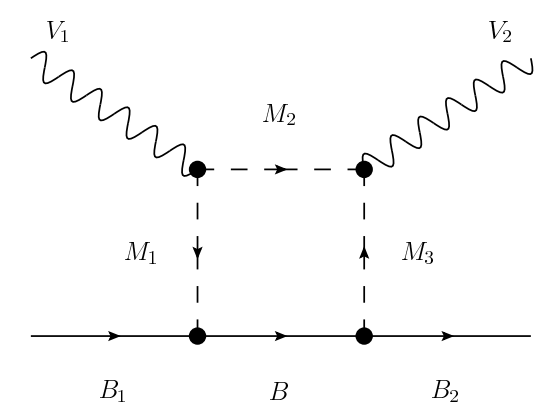

FIG. 2: Diagram for the $V B \rightarrow V B$ interaction incorporating the intermediate pseudoscalar-baryon states.

In the intermediate $B$ states of Fig. 2 we include baryons of the octet and the decuplet. The results of the calculations are a small shift and a broadening of the resonances compared with what is obtained with the basis of vector-baryon alone. 


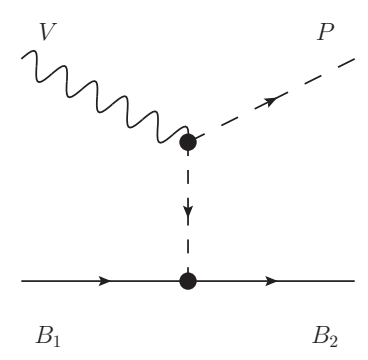

$(a)$

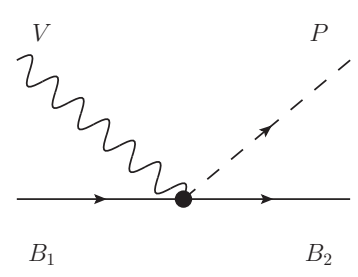

$(b)$

FIG. 3: Diagrams of the $V B \rightarrow P B$. (a) meson pole term, (b) Kroll-Ruderman contact term.

In Fig. 4 we observe two peaks for the states having quantum numbers $S=0$ and $I=1 / 2$, one around $1700 \mathrm{MeV}$, in channels $\rho N$ and $K^{*} \Lambda$, and another peak near $1980 \mathrm{MeV}$, which appears in all the channels except for $\rho N$. It can be seen that the mixing of the $P B$ channels (solid lines) affects differently the two spin sectors, $J^{P}=1 / 2^{-}$and $3 / 2^{-}$, as a consequence of the extra mechanisms contributing mainly to the $J^{P}=1 / 2^{-}$. Indeed, the $P B-V B$ mixing mechanism is more important in the $J^{P}=1 / 2^{-}$sector because the Kroll-Ruderman term only allows the $1 / 2^{-}$pseudoscalar baryon intermediate states in the box diagram. The most important feature is a breaking of the degeneracy for the peak around $1700 \mathrm{MeV}$. This is most welcome since allows us to associate the $1 / 2^{-}$peak found at $1650 \mathrm{MeV}$ with the $N^{*}(1650)\left(1 / 2^{-}\right)$while the peak for $3 / 2^{-}$at $1700 \mathrm{MeV}$ can be naturally associated to the $N^{*}(1700)\left(3 / 2^{-}\right)$. However, let us recall that in the baryon lines of Fig. 2 we only include ground states $(N$ and $\Delta)$. A resonance like the $N^{*}(1520)\left(3 / 2^{-}\right)$also appears dynamically generated in the scheme extending the space to $\pi N$ (d-wave) and $\pi \Delta(\mathrm{d}$-wave) and will be reported elsewhere [70].

The resonances obtained are summarized in Tables \ and II. There are states which one can easily associate to known resonances, and there remain ambiguities in other cases. The nearly degeneracy in spin that the theory predicts is clearly visible in the experimental data, as one sees a few states with about $50 \mathrm{MeV}$ or less mass difference between them. In some cases, the theory predicts quantum numbers for resonances which have no assigned spin and parity. It would be interesting to pursue the experimental determination of these quantum numbers to test the theoretical predictions. In addition, the predictions made here for resonances not yet observed should be a stimulus for further search of such states. In this sense it is worth noting the experimental program at Jefferson Lab [69] to investigate the $\Xi$ resonances. With admitted uncertainties of about $50 \mathrm{MeV}$ in masses, we are confident that the predictions shown here stand on solid grounds and look forward to progress in the area of baryon spectroscopy and on the understanding of the nature of the baryonic resonances.

Before finishing this section we note that the vector meson-baryon and pseudoscalar-baryon coupled problem has also been studied by taking the s-, t- and u-channel diagrams together with a contact term originating from the hidden local symmetry Lagrangian to obtain the $V B$ interaction 67. The $P B$ interaction was calculated using the Kroll-Ruderman contact term, which contrary to [64], refrained the authors from investigating the possible effects of coupling $P B$ to $V B$ channels with spin $3 / 2$. In the spin $1 / 2$ case, some new resonances were found to get generated by the coupled $P B$ and $V B$ dynamics [65, 66, 68]. The coupling constants of the low-lying resonances to the $V B$ channels were also obtained in Ref. [65, 68], which can be useful in studying the photoproduction of these states. 

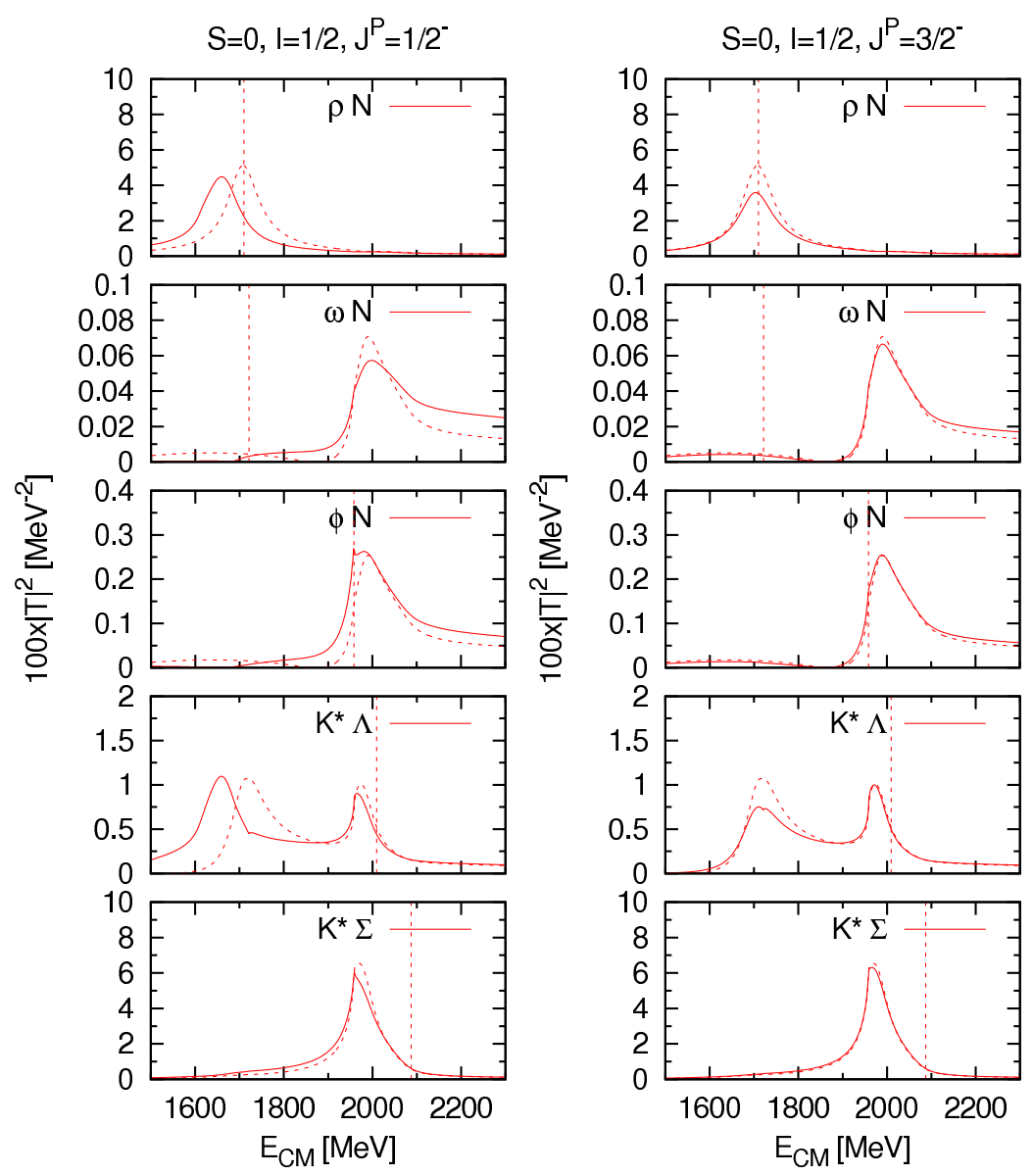

FIG. 4: $|T|^{2}$ for the $\mathrm{S}=0, \mathrm{I}=1 / 2$ states. Dashed lines correspond to tree level only and solid lines are calculated including the box diagram potential. Vertical dashed lines indicate the channel threshold.

\section{HIDDEN CHARM BARYONS FROM VECTOR-BARYON INTERACTION}

Following the idea of [47] it was found in [7] that several baryon states emerged as hidden charm composite states of mesons and baryons with charm. In particular, in the context of vector-baryon interaction, a hidden charm baryon that couples to $J / \psi N$ and other related channels was found, as shown in Tables III and IV. This will play a role later on when we discuss the $J / \psi$ suppression in nuclei. The calculations in the charm sector require an extension to $\mathrm{SU}(4)$ of the hidden gauge Lagrangians, but the symmetry is explicitly broken by using the physical masses of the hadrons involved in the processes. Therefore, when the exchange of a heavy vector meson is implied, the appropriate reduction in the Feynman diagram is taken into account.

In section VI we report on the study of $J / \psi$ propagation in nuclei.

\section{THE PROPERTIES OF $K^{*}$ IN NUCLEI}

Much work about the vector mesons $\rho, \phi, \omega$ in nuclei has been done looking for dileptons 14, 15, 72 74]. Maybe this technical detail is what has prevented any attention being directed to the renormalization of the $K^{*}$ in nuclei. However, recently this problem has been addressed in [75] with very interesting results. 


\begin{tabular}{c|c|cc|ccccc}
\hline \hline$S, I$ & \multicolumn{4}{|c|}{ Theory } & \multicolumn{5}{c}{ PDG data } \\
\hline & pole position & \multicolumn{2}{c|}{ real axis } & \multicolumn{5}{c}{} \\
& $M_{R}+i \Gamma / 2$ & mass & width & name & $J^{P}$ & status & mass & width \\
\hline $0,1 / 2$ & $1690+i 24^{*}$ & 1658 & 98 & $N(1650)$ & $1 / 2^{-}$ & $\star \star \star \star$ & $1645-1670$ & $145-185$ \\
& $1979+i 67$ & 1973 & 85 & $N(2090)$ & $1 / 2^{-}$ & $\star$ & $\approx 2090$ & $100-400$ \\
\hline$-1,0$ & $1776+i 39$ & 1747 & 94 & $\Lambda(1800)$ & $1 / 2^{-}$ & $\star \star \star$ & $1720-1850$ & $200-400$ \\
& $1906+i 34^{*}$ & 1890 & 93 & $\Lambda(2000)$ & $?^{?}$ & $\star$ & $\approx 2000$ & $73-240$ \\
& $2163+i 37$ & 2149 & 61 & & & & & \\
\hline$-1,1$ & - & 1829 & 84 & $\Sigma(1750)$ & $1 / 2^{-}$ & $\star \star \star$ & $1730-1800$ & $60-160$ \\
& - & 2116 & $200-240$ & $\Sigma(2000)$ & $1 / 2^{-}$ & $\star$ & $\approx 2000$ & $100-450$ \\
\hline$-2,1 / 2$ & $2047+i 19^{*}$ & 2039 & 70 & $\Xi(1950)$ & $?^{?}$ & $\star \star \star$ & $1950 \pm 15$ & $60 \pm 20$ \\
& - & 2084 & 53 & $\Xi(2120)$ & $?^{?}$ & $\star$ & $\approx 2120$ & 25 \\
\hline \hline
\end{tabular}

TABLE I: The properties of the nine dynamically generated resonances and their possible PDG counterparts for $J^{P}=1 / 2^{-}$. The numbers with asterisk in the imaginary part of the pole position are obtained without convoluting with the vector mass distribution of the $\rho$ and $K^{*}$.

\begin{tabular}{c|c|cc|ccccc}
\hline \hline$S, I$ & \multicolumn{3}{|c|}{ Theory } & \multicolumn{5}{c}{ PDG data } \\
\hline & pole position & real axis & \multicolumn{5}{c}{} \\
& $M_{R}+i \Gamma / 2$ & mass width & name & $J^{P}$ & status & mass & width \\
\hline $0,1 / 2$ & $1703+i 4^{*}$ & 1705 & 103 & $N(1700)$ & $3 / 2^{-}$ & $\star \star \star$ & $1650-1750$ & $50-150$ \\
& $1979+i 56$ & 1975 & 72 & $N(2080)$ & $3 / 2^{-}$ & $\star \star$ & $\approx 2080$ & $180-450$ \\
\hline$-1,0$ & $1786+i 11$ & 1785 & 19 & $\Lambda(1690)$ & $3 / 2^{-}$ & $\star \star \star$ & $1685-1695$ & $50-70$ \\
& $1916+i 13^{*}$ & 1914 & 59 & $\Lambda(2000)$ & $?^{?}$ & $\star$ & $\approx 2000$ & $73-240$ \\
& $2161+i 17$ & 2158 & 29 & & & & & \\
\hline$-1,1$ & - & 1839 & 58 & $\Sigma(1940)$ & $3 / 2^{-}$ & $\star \star$ & $1900-1950$ & $150-300$ \\
& - & 2081 & 270 & & & & & \\
\hline$-2,1 / 2$ & $2044+i 12^{*}$ & 2040 & 53 & $\Xi(1950)$ & $?^{?}$ & $\star \star \star$ & $1950 \pm 15$ & $60 \pm 20$ \\
& $2082+i 5^{*}$ & 2082 & 32 & $\Xi(2120)$ & $?^{?}$ & $\star$ & $\approx 2120$ & 25 \\
\hline \hline
\end{tabular}

TABLE II: The properties of the nine dynamically generated resonances and their possible PDG counterparts for $J^{P}=3 / 2^{-}$. The numbers with asterisk in the imaginary part of the pole position are obtained without convoluting with the vector mass distribution of the $\rho$ and $K^{*}$.

The $K^{*-}$ width in vacuum is determined in 75 ] by evaluating the imaginary part of the free $\bar{K}^{*}$ self-energy at rest, $\operatorname{Im} \Pi_{\bar{K}^{*}}^{0}$, due to the decay of the $\bar{K}^{*}$ meson into $\bar{K} \pi$ pairs, using the model parameters of the Lagrangians described in Sect. II] The obtained width, $\Gamma_{K^{*-}}=-\operatorname{Im} \Pi_{\bar{K}^{*}}^{0} / m_{\bar{K}^{*}}=$ $42 \mathrm{MeV}$, is quite close to the experimental value of $50.8 \pm 0.9 \mathrm{MeV}$.

The $\bar{K}^{*}$ self-energy in matter, on one hand, results from its decay into $\bar{K} \pi, \Pi_{\bar{K}^{*}}^{\rho, \text { (a) }}$, including both the self-energy of the antikaon [76] and the pion [77, 78] (see first diagram of Fig. [5]and some specific contributions in diagrams $(a 1)$ and $(a 2)$ of Fig. 66). Moreover, vertex corrections required by gauge invariance are also incorporated, and they are associated to the last three diagrams in Fig. 5. Another contribution to the $\bar{K}^{*}$ self-energy comes from its interaction with the nucleons in the Fermi sea, as displayed in diagram (b) of Fig. 6. This accounts for the direct quasi-elastic process $\bar{K}^{*} N \rightarrow \bar{K}^{*} N$, as well as other absorption channels $\bar{K}^{*} N \rightarrow \rho Y, \omega Y, \phi Y, \ldots$ with $Y=\Lambda, \Sigma$. This contribution is determined by integrating the medium-modified $\bar{K}^{*} N$ amplitudes, $T_{\bar{K}^{*} N}^{\rho, I}$, over 


\begin{tabular}{cccccc}
\hline$(I, S)$ & $z_{R}$ & \multicolumn{3}{c}{$g_{a}$} \\
\hline$(1 / 2,0)$ & & $\bar{D}^{*} \Sigma_{c}$ & $\bar{D}^{*} \Lambda_{c}^{+}$ & $J / \psi N$ & \\
& $4415-9.5 i$ & $2.83-0.19 i$ & $-0.07+0.05 i$ & $-0.85+0.02 i$ & \\
& & 2.83 & 0.08 & 0.85 & \\
\hline$(0,-1)$ & & $\bar{D}_{s}^{*} \Lambda_{c}^{+}$ & $\bar{D}^{*} \Xi_{c}$ & $\bar{D}^{*} \Xi_{c}^{\prime}$ & $J / \psi \Lambda$ \\
& $4368-2.8 i$ & $1.27-0.04 i$ & $3.16-0.02 i$ & $-0.10+0.13 i$ & $0.47+0.04 i$ \\
& & 1.27 & 3.16 & 0.16 & 0.47 \\
& $4547-6.4 i$ & $0.01+0.004 i$ & $0.05-0.02 i$ & $2.61-0.13 i$ & $-0.61-0.06 i$ \\
& & 0.01 & 0.05 & 2.61 & 0.61 \\
\hline
\end{tabular}

TABLE III: Pole position $\left(z_{R}\right)$ and coupling constants $\left(g_{a}\right)$ to various channels for the states from $V B \rightarrow V B$ including the $J / \psi N$ and $J / \psi \Lambda$ channels.

\begin{tabular}{ccccc}
\hline$(I, S)$ & $z_{R}$ & \multicolumn{2}{c}{ Real axis } & $\Gamma_{i}$ \\
& & $M$ & $\Gamma$ & \\
\hline$(1 / 2,0)$ & & & & $J / \psi N$ \\
& $4415-9.5 i$ & 4412 & 47.3 & 19.2 \\
\hline$(0,-1)$ & & & & $J / \psi \Lambda$ \\
& $4368-2.8 i$ & 4368 & 28.0 & 5.4 \\
& $4547-6.4 i$ & 4544 & 36.6 & 13.8 \\
\hline
\end{tabular}

TABLE IV: Pole position $\left(z_{R}\right)$, mass $(M)$, total width $(\Gamma$, including the contribution from the light meson and baryon channel) and the decay widths for the $J / \psi N$ and $J / \psi \Lambda$ channels $\left(\Gamma_{i}\right)$. The units are in MeV.

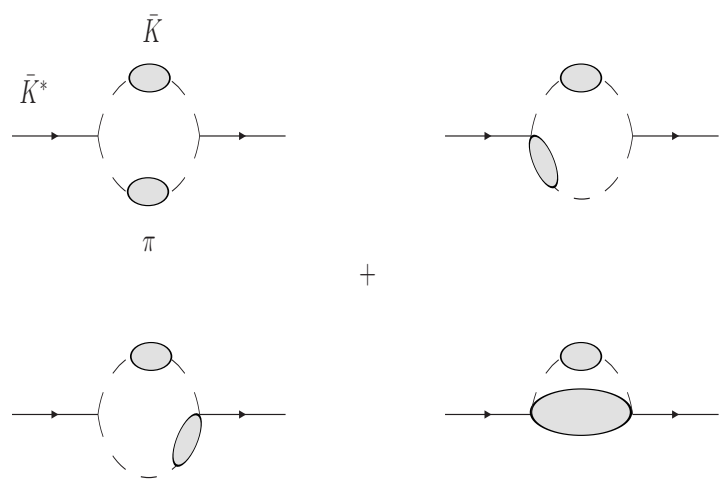

FIG. 5: Self-energy diagrams from the decay of the $\bar{K}^{*}$ meson in the medium.

the Fermi sea of nucleons and, therefore, it will be sensitive to the resonant structures in these amplitudes. In particular, the in-medium amplitudes retain clear traces of two resonances that are generated from the $\bar{K}^{*} N$ interaction and related channels at $1783 \mathrm{MeV}$ and $1830 \mathrm{MeV}$ [47], which can be identified with the experimentally observed $J^{P}=1 / 2^{-}$states $\Lambda(1800)$ and $\Sigma(1750)$, respectively. Note that the self-energy $\Pi_{\bar{K}^{*}}^{\rho,(\mathrm{b})}$ has to be determined self-consistently since it is obtained from the in-medium amplitude $T_{\bar{K}^{*} N}^{\rho}$ which contains the $\bar{K}^{*} N$ loop function $G_{\bar{K}^{*} N}^{\rho}$, and this last quantity itself is a function of the complete self-energy $\Pi_{\bar{K}^{*}}^{\rho}=\Pi_{\bar{K}^{*}}^{\rho \text { (a) }}+\Pi_{\bar{K}^{*}}^{\rho,(\mathrm{b})}$.

The two contributions to the $\bar{K}^{*}$ self-energy, coming from the decay of $\bar{K} \pi$ pairs in the medium 


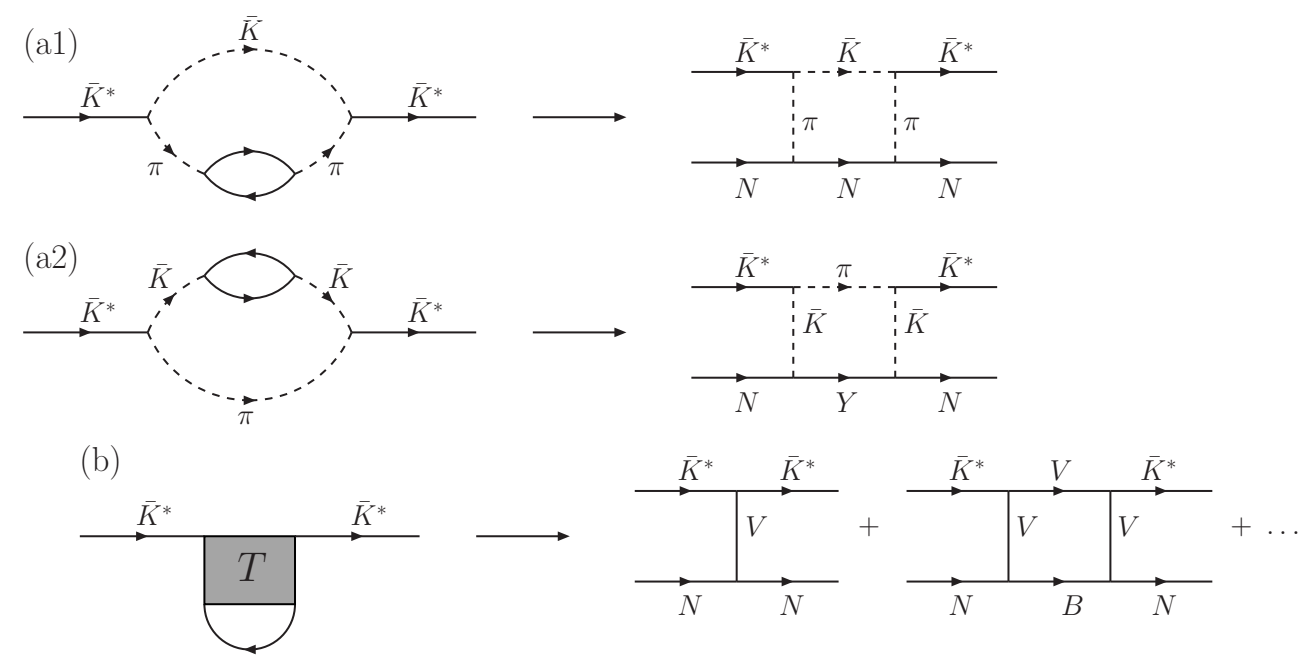

FIG. 6: Contributions to the $\bar{K}^{*}$ self-energy, depicting their different inelastic sources.

[Figs. 6(a1) and 6(a2)] or from the $\bar{K}^{*} N$ interaction [Fig. 6(b)] provide different sources of inelastic $\bar{K}^{*} N$ scattering, which add incoherently in the $\bar{K}^{*}$ width. Note that the $\bar{K}^{*} N$ amplitudes mediated by intermediate $\bar{K} N$ or $\pi Y$ states are not unitarized, in contrast to what is done for the contributions from intermediate $V B$ states. The problem arises because the exchanged pion may be placed on its mass shell, which forces one to keep track of the proper analytical cuts making the iterative process more complicated. A technical solution can be found by calculating the box diagrams of Figs. 6(a1) and 6(a2), taking all the cuts into account properly, and adding the resulting $\bar{K}^{*} N \rightarrow \bar{K}^{*} N$ terms to the $V B \rightarrow V^{\prime} B^{\prime}$ potential coming from vector-meson exchange, in a similar way as done for the study of the vector-vector interaction in Refs. [79, 80]. As we saw in the former sections, the generated resonances barely change their position for spin $3 / 2$ and only by a moderate amount in some cases for spin $1 / 2$. Their widths are somewhat enhanced due to the opening of the newly allowed $P B$ decay channels [64].

The full $\bar{K}^{*}$ self-energy as a function of the $\bar{K}^{*}$ energy for zero momentum at normal nuclear matter density is shown in Fig. 7. We explicitly indicate the contribution to the self-energy coming from the self-consistent calculation of the $\bar{K}^{*} N$ effective interaction (dashed lines) and the selfenergy from the $\bar{K}^{*} \rightarrow \bar{K} \pi$ decay mechanism (dot-dashed lines), as well as the combined result from both sources (solid lines). Around $q^{0}=800-900 \mathrm{MeV}$ we observe an enhancement of the width as well as some structures in the real part of the $\bar{K}^{*}$ self-energy. The origin of these structures can be traced back to the coupling of the $\bar{K}^{*}$ to the in-medium $\Lambda(1783) N^{-1}$ and $\Sigma(1830) N^{-1}$ excitations, which dominate the $\bar{K}^{*}$ self-energy in this energy region. However, at lower energies where the $\bar{K}^{*} N \rightarrow V B$ channels are closed, or at large energies beyond the resonance-hole excitations, the width of the $\bar{K}^{*}$ is governed by the $\bar{K} \pi$ decay mechanism in dense matter.

As we can see, around $q^{0}=m_{\bar{K}^{*}}$ the $\bar{K}^{*}$ feels a moderately attractive optical potential and acquires a width of $260 \mathrm{MeV}$, which is about five times its width in vacuum. A method to measure this large width experimentally was devised in [75] suggesting to use the transparency ratio defined as

$$
T_{A}=\frac{\tilde{T}_{A}}{\tilde{T}_{12} C} \quad, \text { with } \tilde{T}_{A}=\frac{\sigma_{\gamma A \rightarrow K^{+} K^{*-} A^{\prime}}}{A \sigma_{\gamma N \rightarrow K^{+} K^{*-} N}} .
$$

The quantity $\tilde{T}_{A}$ is the ratio of the nuclear $K^{*-}$-photoproduction cross section divided by $A$ times the same quantity on a free nucleon. This describes the loss of flux of $K^{*-}$ mesons in the nucleus 


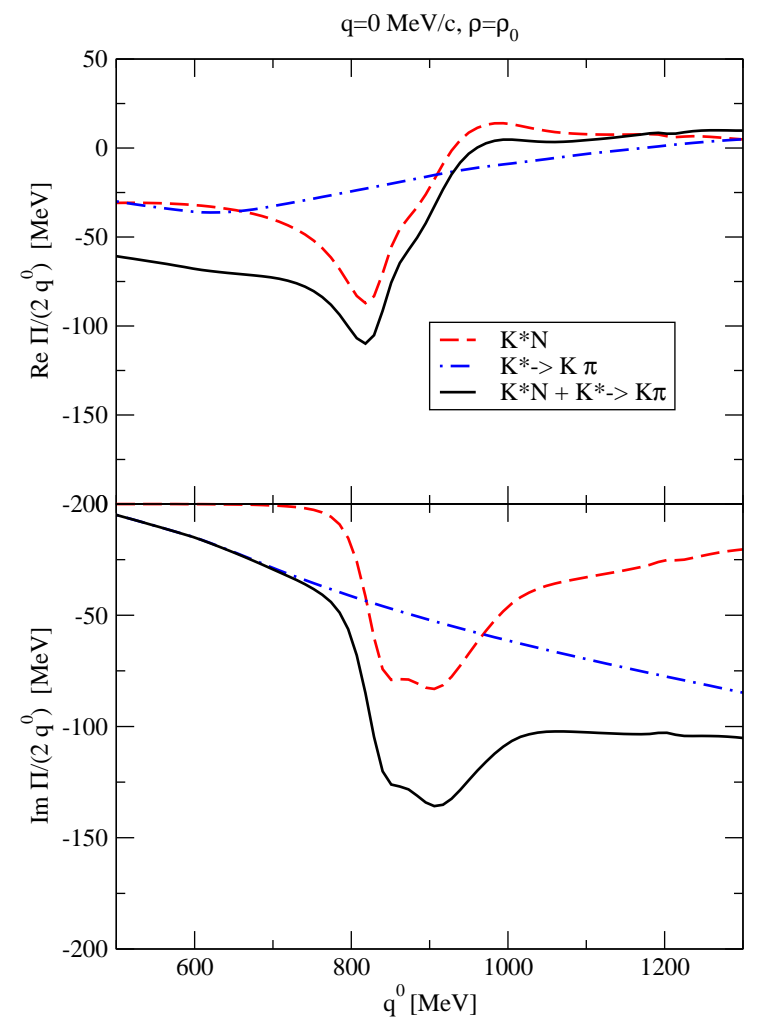

FIG. 7: $\quad \bar{K}^{*}$ self-energy for $\vec{q}=0 \mathrm{MeV} / \mathrm{c}$ and $\rho_{0}$.

and is related to the absorptive part of the $K^{*-}$-nucleus optical potential and, therefore, to the $K^{*-}$ width in the nuclear medium. In order to remove other nuclear effects not related to the absorption of the $K^{*-}$, it was also suggested to look at this ratio with respect to that of a medium nucleus like ${ }^{12} \mathrm{C}, T_{A}$ (see [81]). Results for $T_{A}$ as a function of $A$ can be seen in [5], indicating a sizable depletion of $\bar{K}^{*}$ production in nuclei that we would like to encourage to be measured.

\section{VI. $J / \psi$ SUPPRESSION}

$J / \psi$ suppression in nuclei has been a hot topic [82], among other reasons for its possible interpretation as a signature of the formation of quark gluon plasma in heavy ion reactions [83], but many other interpretations have been offered [84 86]. In a recent paper [87] a study has been done of different $J / \psi N$ reactions which lead to $J / \psi$ absorption in nuclei. The different reactions considered are the transition of $J / \psi N$ to $V N$ with $V$ being a light vector, $\rho, \omega, \phi$, together with the inelastic channels, $J / \psi N \rightarrow \bar{D} \Lambda_{c}$ and $J / \psi N \rightarrow \bar{D} \Sigma_{c}$. Analogously, the mechanisms where an exchanged $D$ collides with a nucleon giving rise to $\pi \Lambda_{c}$ or $\pi \Sigma_{c}$ states are also considered.

The total, elastic and inelastic cross sections, obtained from the unitarized $J / \psi N \rightarrow J / \psi N$ amplitude where only intermediate vector-baryon states are considered, are shown in Fig. 8, We can clearly see the peak around $4415 \mathrm{MeV}$ produced by the hidden charm resonance, described in Tables III and IV] dynamically generated from the interaction of $J / \psi N$ with its coupled $V B$ channels.

The cross sections for the inelastic transitions $J / \psi N \rightarrow \bar{D} \Lambda_{c}$ and $J / \psi N \rightarrow \bar{D} \Sigma_{c}$ are shown in Fig. 9. We can see that the first cross section is sizable and bigger than the one from the 


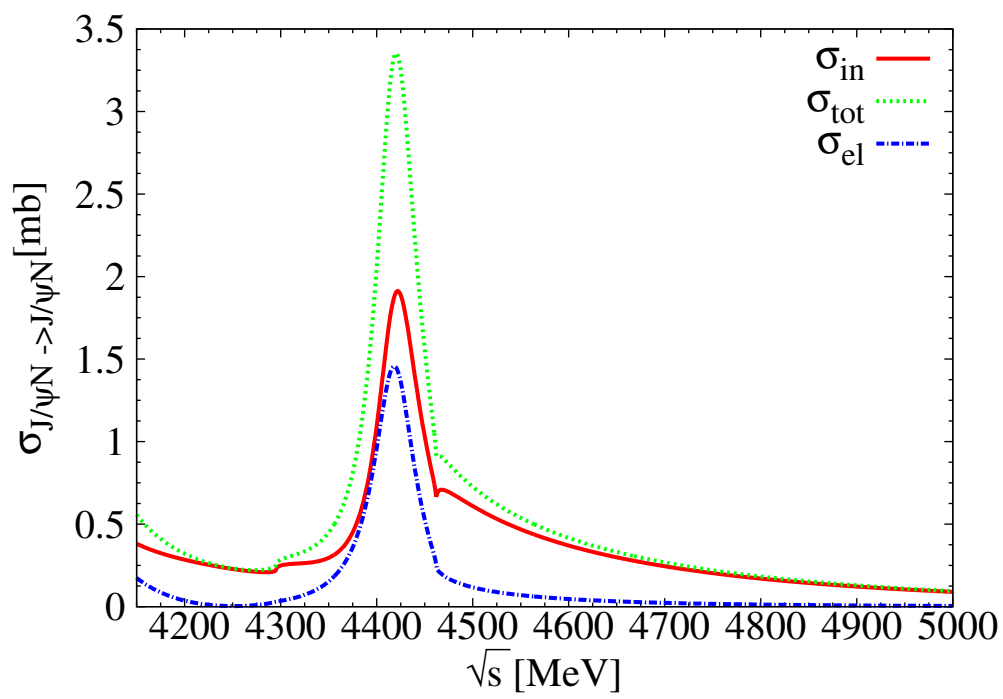

FIG. 8: The total, elastic and inelastic cross sections obtained from the unitary $J / \psi N \rightarrow J / \psi N$ amplitude involving only intermediate vector-baryon states.
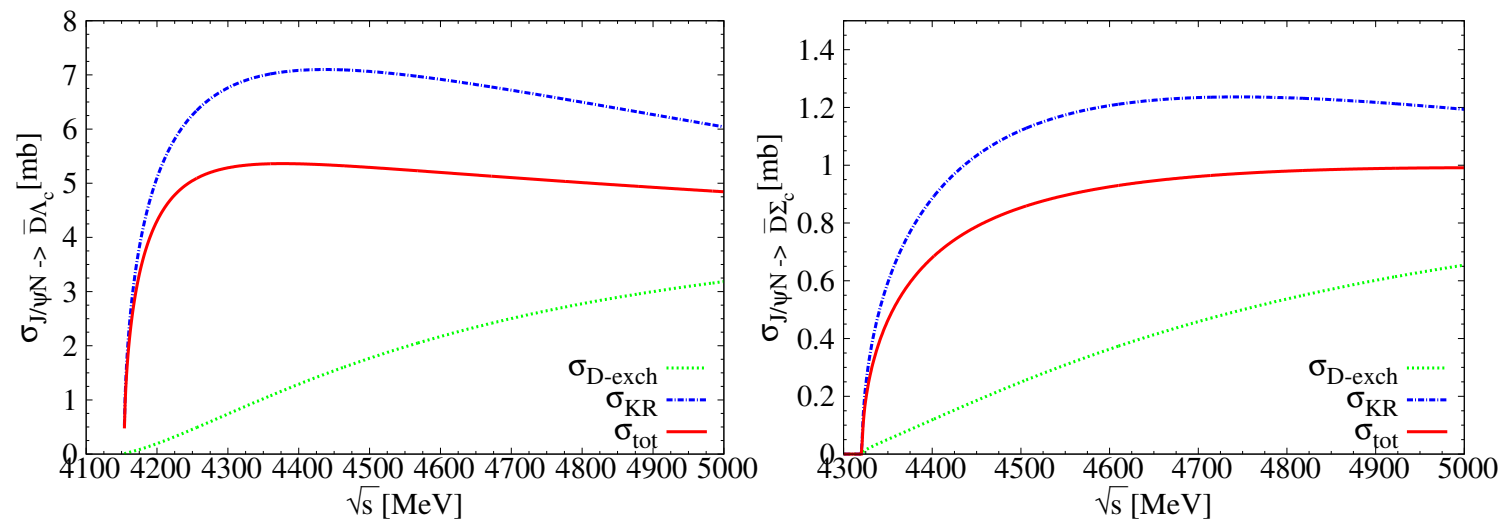

FIG. 9: The cross section for $J / \psi N \rightarrow \bar{D} \Lambda_{c}$ (up) and $J / \psi N \rightarrow \bar{D} \Sigma_{c}$ (down).

$V B$ channels. The cross sections for $J / \psi N \rightarrow \bar{D} \pi \Lambda_{c}$ or $\bar{D} \pi \Sigma_{c}$ are small in size in the region of interest and are not plotted here. The total $J / \psi N$ inelastic cross section, obtained as the sum of all inelastic cross sections from the different sources discussed before, is represented in Fig. 10. (left).

With the inelastic cross section obtained, the transparency ratio for electron induced $J / \psi$ production in nuclei at beam energies around $10 \mathrm{GeV}$ has been studied. The results are shown in Fig. 10 (right) where the transparency ratio of ${ }^{208} \mathrm{~Pb}$ relative to that of ${ }^{12} \mathrm{C}$ is displayed as a function of the energy. It is clear that one finds sizable reductions in the rate of $J / \psi$ production in electron induced reactions. It should be noted that the calculation of the transparency ratio discussed so far does not consider the shadowing of the photons and assumes they can reach every point without being absorbed. However, for $\gamma$ energies of around $10 \mathrm{GeV}$, as suggested here, the photon shadowing, or initial photon absorption, cannot be ignored. Taking this into account is easy since one must multiply the ratio $T_{A}$ by the ratio of $N_{\text {rmeff }}$ for a nucleus of mass $A$ relative to ${ }^{12} \mathrm{C}$. This ratio for ${ }^{208} \mathrm{~Pb}$ to ${ }^{12} \mathrm{C}$ at $E_{\gamma}=10 \mathrm{GeV}$ is of the order 0.8 , but with uncertainties [88]. This factor 
is applied to the lower curve of Fig. 10 (right) for a proper comparison with experiment. The results for the transparency ratio imply that $30-35 \%$ of the $J / \psi$ produced in heavy nuclei are absorbed inside the nucleus. This is very much in line with depletions of $J / \psi$ in matter observed in other reactions and offers another perspective in the interpretation of the $J / \psi$ suppression in terms of hadronic reactions, which has also been advocated before [86]. Apart from novelties in the details of the calculations and the reaction channels considered, we find that the presence of the resonance that couples to $J / \psi N$ produces a peak in the inelastic $J / \psi N$ cross section and a dip in the transparency ratio. However, this dip is washed away when effects of Fermi motion are taken into account.
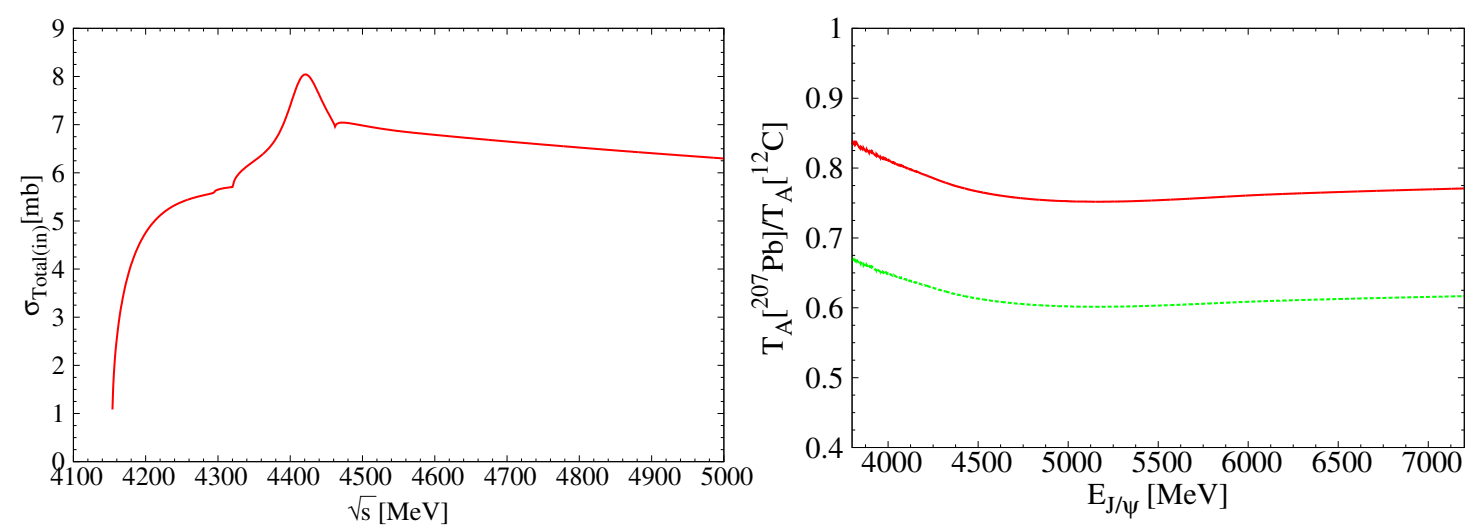

FIG. 10: Left: The total inelastic cross section of $J / \psi N$. Right: The transparency ratio of $J / \psi$ photoproduction as a function of the energy in the $\mathrm{CM}$ of $J / \psi$ with nucleons of the nucleus. Solid line: represents the effects due to $J / \psi$ absorption. Dashed line: includes photon shadowing [88].

\section{CONCLUSIONS}

We have made a survey of recent developments along the interaction of vector mesons with baryons and the properties of some vector mesons in a nuclear medium. We showed that the interaction is strong enough to produce resonant states which can qualify as quasibound states of a vector meson and a baryon in coupled channels. This adds to the wealth of composite states already established from the interaction of pseudoscalar mesons with baryons. At the same time we reported on studies of the mixing of the pseudoscalar-baryon states with the vector-baryon states which break the spin degeneracy that the original model had. The mechanisms of vector-baryon interaction extended to the charm sector also produced some hidden charm states which couple to the $J / \psi N$ channel and had some repercussion in the $J / \psi$ suppression in nuclei. We also showed results for the spectacular renormalization of the $K^{*}$ in nuclei, where the width becomes as large as $250 \mathrm{MeV}$ at normal nuclear matter density and we made suggestions of experiments that could test this large change.

\section{Acknowledgments}

This work is partly supported by DGICYT contract numbers FIS2011-28853-C02-01, FIS201124154, the Generalitat Valenciana in the program Prometeo, 2009/090 and Grant No. 2009SGR1289 from Generalitat de Catalunya. L.T. acknowledges support from Ramon y Cajal Research 
Programme, and from FP7-PEOPLE-2011-CIG under contract PCIG09-GA-2011-291679. We acknowledge the support of the European Community-Research Infrastructure Integrating Activity Study of Strongly Interacting Matter (acronym HadronPhysics3, Grant Agreement n. 283286) under the Seventh Framework Programme of EU.

[1] R. Rapp and J. Wambach, Adv. Nucl. Phys. 25 (2000) 1

[2] R. S. Hayano and T. Hatsuda, Rev. Mod. Phys. 82, 2949 (2010)

[3] S. Leupold, V. Metag and U. Mosel, Int. J. Mod. Phys. E 19, 147 (2010)

[4] M. Bando, T. Kugo, S. Uehara, K. Yamawaki and T. Yanagida, Phys. Rev. Lett. 54, 1215 (1985).

[5] M. Bando, T. Kugo and K. Yamawaki, Phys. Rept. 164, 217 (1988).

[6] U. G. Meissner, Phys. Rept. 161, 213 (1988).

[7] R. Rapp, G. Chanfray and J. Wambach, Nucl. Phys. A 617, 472 (1997)

[8] W. Peters, M. Post, H. Lenske, S. Leupold and U. Mosel, Nucl. Phys. A 632, 109 (1998)

[9] R. Rapp and J. Wambach, Adv. Nucl. Phys. 25, 1 (2000)

[10] M. Urban, M. Buballa, R. Rapp and J. Wambach, Nucl. Phys. A 673, 357 (2000)

[11] D. Cabrera, E. Oset and M. J. Vicente Vacas, Nucl. Phys. A 705, 90 (2002)

[12] D. Cabrera and M. J. Vicente Vacas, Phys. Rev. C 67, 045203 (2003)

[13] G. E. Brown and M. Rho, Phys. Rev. Lett. 66, 2720 (1991).

[14] S. Damjanovic et al. [NA60 Collaboration], Nucl. Phys. A 783 (2007) 327

[15] N.H. Wood et al. [CLAS Collaboration], Phys. Rev. C 78 (2008) 015201

[16] D. Trnka et al. [CBELSA/TAPS Collaboration], Phys. Rev. Lett. 94, 192303 (2005)

[17] M. Kaskulov, E. Hernandez and E. Oset, Eur. Phys. J. A 31, 245 (2007)

[18] M. Nanova et al. [CBELSA/TAPS Collaboration], Phys. Rev. C 82, 035209 (2010)

[19] D. Trnka, PhD Thesis, University of Giessen (Germany).

[20] V. Metag, Prog. Theor. Phys. Suppl. 168, 503 (2007).

[21] M. Kaskulov, H. Nagahiro, S. Hirenzaki and E. Oset, Phys. Rev. C 75, 064616 (2007)

[22] V. Metag, Prog. Part. Nucl. Phys. 61, 245 (2008)

[23] M. Kotulla et al. [CBELSA/TAPS Collaboration], Phys. Rev. Lett. 100, 192302 (2008)

[24] M. Kaskulov, E. Hernandez and E. Oset, Eur. Phys. J. A 46, 223 (2010)

[25] N. Kaiser, P. B. Siegel and W. Weise, Phys. Lett. B 362, 23 (1995)

[26] E. Oset and A. Ramos, Nucl. Phys. A 635 (1998) 99

[27] J. A. Oller and U. G. Meissner, Phys. Lett. B 500, 263 (2001)

[28] C. Garcia-Recio, J. Nieves and L. L. Salcedo, Phys. Rev. D 74 (2006) 034025

[29] T. Hyodo, S. I. Nam, D. Jido and A. Hosaka, Phys. Rev. C 68, 018201 (2003)

[30] Y. Ikeda, T. Hyodo and W. Weise, Nucl. Phys. A 881, 98 (2012) [arXiv:1201.6549 [nucl-th]].

[31] D. Jido, J. A. Oller, E. Oset, A. Ramos and U. G. Meissner, Nucl. Phys. A 725, 181 (2003)

[32] B. Borasoy, R. Nissler and W. Weise, Eur. Phys. J. A 25, 79 (2005)

[33] J. A. Oller, J. Prades and M. Verbeni, Phys. Rev. Lett. 95, 172502 (2005)

[34] J. A. Oller, Eur. Phys. J. A 28, 63 (2006)

[35] B. Borasoy, U. G. Meissner and R. Nissler, Phys. Rev. C 74, 055201 (2006)

[36] T. Hyodo, D. Jido and A. Hosaka, Phys. Rev. C 78, 025203 (2008)

[37] L. Roca, T. Hyodo and D. Jido, Nucl. Phys. A 809, 65 (2008)

[38] V. K. Magas, E. Oset and A. Ramos, Phys. Rev. Lett. 95, 052301 (2005)

[39] D. Jido, E. Oset and T. Sekihara, Eur. Phys. J. A 42, 257 (2009)

[40] A. Martinez Torres, K. P. Khemchandani and E. Oset, Phys. Rev. C 77, 042203 (2008)

[41] A. Martinez Torres, K. P. Khemchandani and E. Oset, Eur. Phys. J. A 35, 295 (2008)

[42] K. P. Khemchandani, A. Martinez Torres and E. Oset, Eur. Phys. J. A 37, 233 (2008)

[43] D. Jido and Y. Kanada-En'yo, Phys. Rev. C 78, 035203 (2008).

[44] Y. Kanada-En'yo and D. Jido, Phys. Rev. C 78, 025212 (2008)

[45] P. Gonzalez, E. Oset and J. Vijande, Phys. Rev. C79, 025209 (2009)

[46] S. Sarkar, B. -X. Sun, E. Oset et al., Eur. Phys. J. A44, 431-443 (2010).

[47] E. Oset, A. Ramos, Eur. Phys. J. A44, 445-454 (2010). 
[48] D. Gamermann, C. Garcia-Recio, J. Nieves and L. L. Salcedo, Phys. Rev. D 84 (2011) 056017.

[49] C. Garcia-Recio, J. Nieves and L. L. Salcedo, Phys. Rev. D 74 (2006) 034025.

[50] C. Garcia-Recio, J. Nieves and L. L. Salcedo, Phys. Rev. D 74, 036004 (2006).

[51] H. Toki, C. Garcia-Recio, J. Nieves, Phys. Rev. D77 (2008) 034001.

[52] C. Garcia-Recio, L. S. Geng, J. Nieves and L. L. Salcedo, Phys. Rev. D 83 (2011) 016007.

[53] C. Garcia-Recio, V. K. Magas, T. Mizutani, J. Nieves, A. Ramos, L. L. Salcedo and L. Tolos, Phys. Rev. D 79, 054004 (2009).

[54] D. Gamermann, C. Garcia-Recio, J. Nieves, L. L. Salcedo and L. Tolos, Phys. Rev. D 81, 094016 (2010).

[55] O. Romanets, L. Tolos, C. Garcia-Recio, J. Nieves, L. L. Salcedo and R. G. E. Timmermans, Phys. Rev. D 85 (2012) 114032.

[56] H. Nagahiro, L. Roca, A. Hosaka and E. Oset, Phys. Rev. D 79, 014015 (2009)

[57] F. Klingl, N. Kaiser and W. Weise, Nucl. Phys. A 624 (1997) 527

[58] J. E. Palomar and E. Oset, Nucl. Phys. A 716, 169 (2003)

[59] G. Ecker, Prog. Part. Nucl. Phys. 35 (1995) 1

[60] V. Bernard, N. Kaiser and U. G. Meissner, Int. J. Mod. Phys. E4 (1995) 193

[61] E. E. Jenkins and A. V. Manohar, Phys. Lett. B 259, 353 (1991).

[62] A. Ramos, E. Oset and C. Bennhold, Phys. Rev. Lett. 89, 252001 (2002)

[63] T. Inoue, E. Oset and M. J. Vicente Vacas, Phys. Rev. C 65, 035204 (2002)

[64] E. J. Garzon and E. Oset, Eur. Phys. J. A 48, 5 (2012)

[65] K. P. Khemchandani, A. M. Torres, H. Kaneko, H. Nagahiro and A. Hosaka, arXiv:1202.3840 [nucl-th].

[66] K. P. Khemchandani, A. Martinez Torres, H. Kaneko, H. Nagahiro and A. Hosaka, Phys. Rev. D 84, 094018 (2011)

[67] K. P. Khemchandani, H. Kaneko, H. Nagahiro and A. Hosaka, Phys. Rev. D 83, 114041 (2011)

[68] K. P. Khemchandani, A. Martinez Torres, H. Nagahiro and A. Hosaka, Phys. Rev. D 85, 114020 (2012)

[69] J. W. Price et al. [CLAS Collaboration], Phys. Rev. C 71, 058201 (2005)

[70] E. J. Garzon, Xie J. J. and E. Oset, to be published.

[71] J. -J. Wu, R. Molina, E. Oset and B. S. Zou, Phys. Rev. Lett. 105, 232001 (2010); J. -J. Wu, R. Molina, E. Oset and B. S. Zou, Phys. Rev. C 84, 015202 (2011)

[72] I. Frohlich et al. [HADES Collaboration], Int. J. Mod. Phys. A 24, 317 (2009)

[73] O. Buss, T. Gaitanos, K. Gallmeister, H. van Hees, M. Kaskulov, O. Lalakulich, A. B. Larionov and T. Leitner et al., Phys. Rept. 512, 1 (2012)

[74] M. Naruki, H. Funahashi, Y. Fukao, M. Kitaguchi, M. Ishino, H. Kanda, S. Mihara and T. Miyashita et al., Phys. Rev. Lett. 96, 092301 (2006)

[75] L. Tolos, R. Molina, E. Oset and A. Ramos, Phys. Rev. C 82 (2010) 045210

[76] L. Tolos, A. Ramos and E. Oset, Phys. Rev. C 74 (2006) 015203

[77] E. Oset, P. Fernandez de Cordoba, L.L. Salcedo and R. Brockmann, Phys. Rept. 188 (1990) 79

[78] A. Ramos, E. Oset and L.L. Salcedo, Phys. Rev. C 50 (1994) 2314

[79] R. Molina, D. Nicmorus and E. Oset, Phys. Rev. D 78, 114018 (2008)

[80] L. S. Geng and E. Oset, Phys. Rev. D79 (2009) 074009

[81] V. K. Magas, L. Roca and E. Oset, Phys. Rev. C 71, 065202 (2005)

[82] R. Vogt, Phys. Rept. 310, 197 (1999).

[83] T. Matsui and H. Satz, Phys. Lett. B 178, 416 (1986).

[84] R. Vogt, Nucl. Phys. A 700, 539 (2002)

[85] B. Z. Kopeliovich and B. G. Zakharov, Phys. Rev. D 44, 3466 (1991).

[86] A. Sibirtsev, K. Tsushima and A. W. Thomas, Phys. Rev. C 63, 044906 (2001)

[87] R. Molina, C. W. Xiao and E. Oset, Phys. Rev. C 86, 014604 (2012).

[88] N. Bianchi et al., Phys. Rev. C 54, 1688 (1996). 